\title{
Cancer Stem Cells: Coming of Age
}

\section{Fazlul H Sarkar* and Asfar S Azmi*}

Department of Pathology, Karmanos Cancer Institute, Wayne State University School of Medicine, Detroit MI, USA

The term "stem cell" was proposed in the early 1900s by Russian histologist Alexander Maksimov who postulated the existence of hematopoietic stem cells possessing two important properties, i.e. selfrenewal and differentiation capacity. Since then enormous amount of research on hematopoietic stem cells has been documented which provided important insights into the behavior of stem cells. Such understanding led to the development of therapeutic approaches for different disease conditions. On the contrary, the idea that tumors harbor a sub-set of cells with stem-like cell characteristics and termed as cancer stem cells (CSCs) has been controversial and a hotly debated topic [1]. Till recently, there was no consensus among the researchers over the existence of such sub-type of cells within the tumor mass that may be refractory to chemotherapy/radiotherapy or have a different growth pattern compared to the bulk of the tumor cells in the tumor mass [2]. Such skepticism was due in part to the lack of proper tools and adequate screening capabilities that hindered the proper characterization and isolation of these elusive cells.

The first conclusive evidence for CSCs was published in 1997 when Bonnet and Dick isolated a subpopulation of leukemic cells that expressed a specific surface marker CD34, but lacked the expression of CD38 [3]. The authors have concluded that these CD34 ${ }^{+} / \mathrm{CD} 38^{-}$cells are capable of initiating tumors in a mice model which recapitulated the histologic and other characteristic that was similar to the original tumor. Since then a number of laboratories all across the globe have been able to capture, characterize and propagate CSCs obtained from multiple human tumor types. Recent advancement in both cell characterization methods and isolation techniques have allowed for deeper understanding of tumor heterogeneity and CSCs [4]. Researchers are able to simultaneously sort heterogeneous cell populations for multiple stem cell like markers [5]. These advancements coupled with omics (genomic, proteomic, interactomic and mathematical modeling) tools have rapidly accelerated stem cell research in the past 15 years [6]. It is safe to say that tremendous progress has been made in this direction documenting, to a large extent, led to put the debate surrounding the existence of CSCs to rest.

Cancer stem cells have been proposed to be the major cause for therapeutic resistance in tumors [7]. Generally, most of the chemo, neoadjuvant and targeted therapies are able to target bulk of the tumors. However, it is these refractory CSCs that remain un-touched by the drug treatment, resulting in tumor recurrence. Therefore, prior to designing regimens against heterogeneous tumor cells within a tumor, the CSCs signaling needs to be better understood. The hallmark work of Weinberg and colleagues as well as enhancement in 3D culture techniques has helped in deeper understanding of CSC growth kinetics and phenotype [8]. A collective analysis of works from different laboratories points to a highly complex interaction network involving multiple signaling pathways and cross talk with non-coding RNAs (microRNAs) that in-turn supports the existence and plasticity of CSCs [9]. This allows for their greater motility which renders them to initiate cancers at distant site. Interestingly, reversal of CSCs mesenchymal feature has been suggested to be a major form of therapy against these resistant cells. It is increasingly being recognized that any successful cancer therapy should be designed to target both bulk population of cancer cells in a tumor as well as the stem cell fraction within the tumor
[10]. Stem cells are projected to play pivotal role in cancer diagnostics, selective drug targeting, prevention of metastasis, and development of newer intervention/therapeutic strategies. Nevertheless, progress in CSC research has been slow, and through this thematic issue we take the opportunity to make abreast the research community with the latest developments in this cutting-edge area of research.

We have organized this special issue to touch upon the most current topics in the cancer stem cell research. The topic begins with a highly informative review article by Ivan Gomez and colleagues that details the importance of gene regulatory networks and single cell analysis in the understanding of tumor heterogeneity. Their article is followed by a review from Achuta Guddati who provides detailed analysis of a dual regimen consisting of stem cell antagonist and differentiating agents for successful chemotherapy. In this article the author presents a mathematical model which stimulates stem cell homeostasis and a dual therapy that could successfully eliminate heterogeneous stem cell population. This is followed by our article describing the progress in systems and network pharmacology approaches for the identification and elimination of cancer stem cells. This is followed by three highly informative reviews in which Dr. Adhip Majumdar, Dr. Ahmad and Shadan Ali evaluated the role of cancer stem cells in the progression of colorectal, lung and pancreatic cancer. These multiple reviews provide deeper insights into the cross-talk of multiple pathways along with recently discovered microRNAs. In another important article, Dr. Kiyoko presents modeling data for early carcinogenesis in normal endometrium that was developed through introduction of oncogenic Ras. Finally, Dr. Clarkson, in his review, details the complex role of cancer stem cells in breast cancer heterogeneity and plasticity.

These highly informative reviews are expected to serve as an excellent resource to readers who are interested in pursuing research in the area of cancer stem cells. It was indeed a pleasure to collect this impressive list of articles from experts in the field and we expect enthusiastic readership from the audience that is key to the success of this thematic issue on cancer stem cells.

\section{References}

1. Gupta PB, Chaffer CL, Weinberg RA (2009) Cancer stem cells: mirage or reality?. Nat Med 15: 1010-1012.

*Corresponding authors: Fazlul H. Sarkar, Ph.D. (Distinguished Professor) Department of Pathology, Karmanos Cancer Institute, Wayne State University School of Medicine, Detroit MI, USA, E-mail: fsarkar@med.wayne.edu

Asfar S. Azmi, PhD. (Research Scientist), Department of Pathology, Karmanos Cancer Institute, Wayne State University School of Medicine, Detroit MI, USA E-mail: azmia@karmanos.org

Received December 17, 2012; Accepted December 18, 2012; Published December 20, 2012

Citation: Sarkar FH, Azmi AS (2012) Cancer Stem Cells: Coming of Age. J Stem Cell Res Ther S7:e001. doi:10.4172/2157-7633.S7-e001

Copyright: (C) 2012 Sarkar FH, et al. This is an open-access article distributed under the terms of the Creative Commons Attribution License, which permits unrestricted use, distribution, and reproduction in any medium, provided the original author and source are credited. 
2. Gilbertson RJ, Graham TA (2012) Cancer: Resolving the stem-cell debate. Nature 488: 462-463.

3. Bonnet D, Dick JE (1997) Human acute myeloid leukemia is organized as a hierarchy that originates from a primitive hematopoietic cell. Nat Med 3: 730737.

4. Valent P, Bonnet D, De Maria R, Lapidot T, Copland M, et al. (2012) Cance stem cell definitions and terminology: the devil is in the details. Nat Rev Cancer 12: $767-775$

5. Tosoni D, Di Fiore PP, Pece S (2012) Functional purification of human and mouse mammary stem cells. Methods Mol Biol 916: 59-79.
6. Turner C, Kohandel M (2012) Quantitative approaches to cancer stem cells and epithelial-mesenchymal transition. Semin Cancer Biol 22: 374-378.

7. Delude C (2011) Tumorigenesis: Testing ground for cancer stem cells. Nature 480: S43-S45

8. Mani SA, Guo W, Liao MJ, Eaton EN, Ayyanan A, et al. (2008) The epithelialmesenchymal transition generates cells with properties of stem cells. Cell 133 704-715.

9. Bao B, Azmi AS, Ali S, Ahmad A, Li Y, et al. (2012) The biological kinship of hypoxia with CSC and EMT and their relationship with deregulated expression of miRNAs and tumor aggressiveness. Biochim Biophys Acta 1826: 272-296.

10. Yi SY, Hao YB, Nan KJ, Fan TL (2012) Cancer stem cells niche: A target for novel cancer therapeutics. Cancer Treat Rev.
This article was originally published in a special issue, Cancer Stem Cells handled by Editor(s). Fazlul Hoque Sarkar, Wayne State University, USA; Asfar Sohail Azmi, Wayne State University, USA 